\title{
UN NOUVEL EUGLÉNIEN POLYFLAGELLÉ PARASITE DU TÊTARD DE LEPTODACTYLUS OCELLATUS AU BRÉSIL.
}

\author{
Par E. BRUMPT et G. LAVIER
}

En octobre 1913, l'un de nous eut l'occasion de découvrir dans les environs de São-Paulo (Brésil) plusieurs gîtes à têtards de Leptodactylus ocellatus (Linné). Tous ces têtards hébergeaient dans leur rectum un organisme allongé à aspect d'Euglénien, très métabolique mais ayant en état de repos une longueur d'environ $50 \mu$ sur une largeur moyenne de 5 à $7 \mu$ et présentant alors une extrémité postérieure effilée et une extrémité antérieure légèrement arrondie, avec un appareil pharyngien, un cytoplasme bourré de vacuoles dont une antérieure plus grande, une striation cuticulaire nette, 6 ou 7 flagelles égaux, pas de chlorophylle, pas de stigma; le noyau bien visible occupait une situation médiane.

Des frottis furent faits et les uns, après fixation humide, colorés à l'hématoxylinę ferrique, les autres colorés directement par le May-Grünwald-Giemsa. Les premières préparations, par le montage au baume du Canada, ont fait subir au flagellé une légère rétraction, les autres lui ont conservé très sensiblement ses dimensions normales. Les deux méthodes de coloration donnent d'ailleurs des résultats identiques mais les préparations au Giemsa sont plus fines et les détails y sont plus facilement observables. C'est d'après elles que nous étudierons la structure de cet organisme (fig. 1).

Les dimensions totales sont lès suivantes :

Pour 3 exemplaires colorés à l'hématoxyline ferrique :

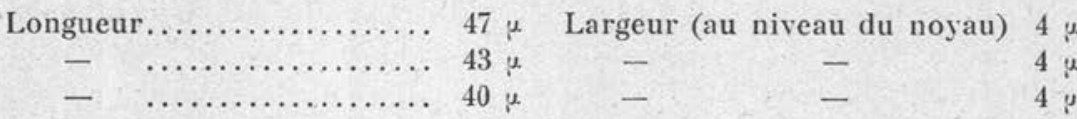

Pour 3 exemplaires colorés au Giemsa :

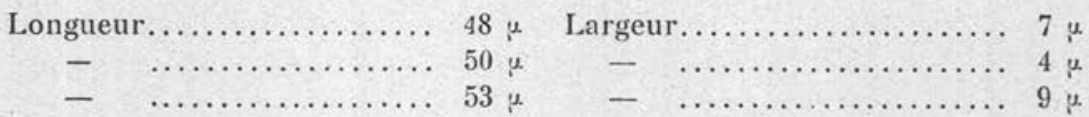

Le cytoplasme est bourré de nombreuses vacuoles ; celles-ci sont de dimensions variables mais ont en moyenne 3 à $5 \mu$ de diamètre.

Annales de Parasitologie, $\mathrm{T}$. II, $\mathrm{N}^{\circ} 3$. - Juillet 1924 , p. 248. 
Entre elles se trouvent de grosses granulations ovoïdes, de $1 \mu$ environ de longueur et fortement basophiles, se colorant intensivement par l'hématoxyline et par le Giemsa ; enfin à l'extrémité antérieure est constamment présente la grande vacuole que nous avons signalée plus haut. Elle est sphérique et mesure 5 à $7 \mu$ de diamètre ; elle est analogue au " réservoir " bien connu des Eugléniens.

Elle communique avec l'extérieur par le pharynx. Celui-ci, d'ailleurs fort court, contient les flagelles et est difficile à voir. Mais dans certains exemplaires (fig. 2, B), on peut distinguer nettement son orifice externe par où les flagellés s'épanouissent en bouquet.

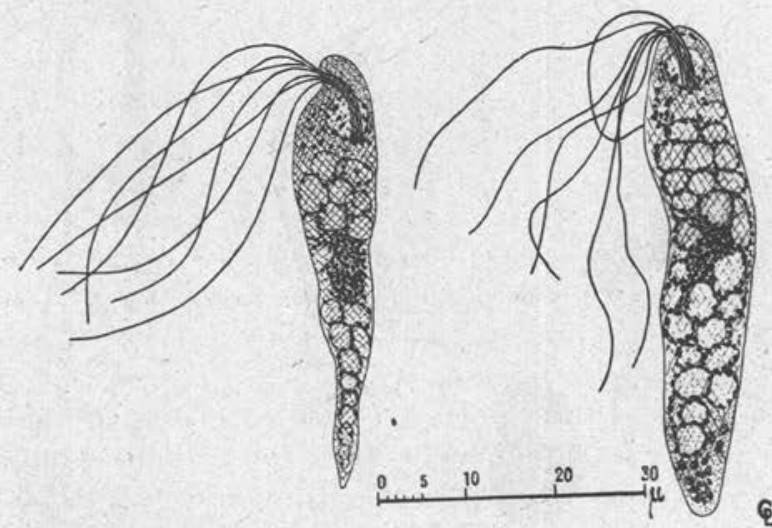

Fig. 1. - Hegneria leptodactyli n. g., n. sp. - Deux exemplaires colorés au May-Grünwald-Giemsa, sur frottis à sec.

Cette bouche n'est pas terminale, mais légèrement subapicale; on peut donc distinguer au flagellé une face dorsale et une face ventrale.

Les flagelles sont au nombre de 7 ; dans quelques rares exemplaires cependant, on n'en rencontre que 6 , et l'aspect intact de ces individus ne permet pas de croire qu'un flagelle ait disparu dans les manipulations de fixation et de coloration. Ces flagelles sont colorés par le Giemsa en rouge pourpre. Ils naissent chacun d'un petit blépharoplaste également coloré en rouge ; il est difficile de voir à la fois les sept blépharoplastes, mais on peut indiscutablement dans chaque individu en voir 4 ou 5 donnant chacun naissance à son flagelle. Ces blépharoplastes sont alignés au niveau du pourtour inférieur de la grande vacuole. Les flagelles traversent cette vacuole où ils sont situés dorsalement quand on les regarde de profil (fig. 2, B), et dans le sens de l'axe quand on regarde la face ventrale (fig. 2, C), ils s'engagent ensuite dans le court pharynx 
et s'épanouissent au sortir de la bouche. Ils sont tous égaux de taille, mesurant 47 à $52 \mu$ dans leur partie libre et 6 à $8 \mu$ dans la partie comprise entre la bouche et les blépharoplastes (partie pharyngovacuolaire).

Le noyau occupe la partie médiane du corps. Il n'a pas de membrane visible et est fortement diffluent; il est formé de nombreuses et grosses granulations sphériques ou ovoïdes formant un amas aú
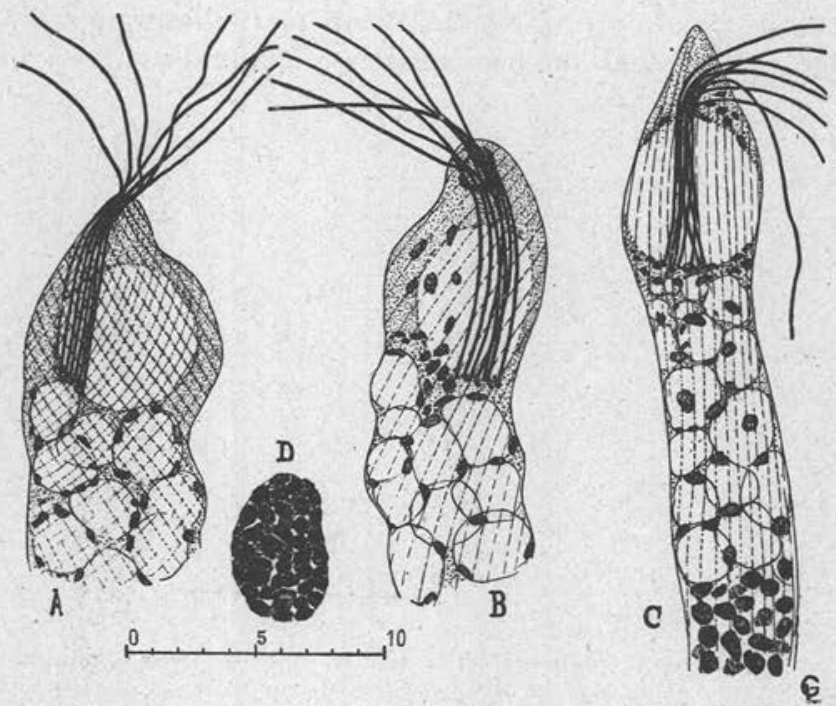

Fıg. 2. - Hegneria leptodactyli n. g., n. sp. - A, Extrémité antérieure d'un individu, vu de trois-quart; la striation cuticulaire paraît quadrillée, les stries de la face postérieure apparaissant derrière celles de la face antérieure qu'elles croisent. B, extrémité antérieure d'un individu montrant nettement la bouche subapicale par où s'échappent les flagelles. C, extrémité antérieure d'un individu vu par la face ventrale. D, noyau d'un individu en état de contraction; le noyau est généralement plus diffluent (voir figure 1).

milieu duquel on distingue assez difficilement un centrosome plus grand, ces granulations ont d'ailleurs un aspect analogue à celles que nous avons signalées dans le cytoplasme. La longueur du noyau est de 7 à $10 \mu$, sa largeur est à peine inférieure de 2 à $3 \mu$ à celle de l'organisme entier.

La cuticule présente une striation oblique fine mais nette; les stries sont pointillées et ont entre elles un intervalle d'environ $0,5 \mu$ (fig. $2, A$ ).

Nous n'avons pas observé dans nos frottis de formes de division.

Ce curieux organisme présente des affinités nettes avec les Euglé- 
niéns dépourvus de chromatophore : mobilité, cuticule ornementée, réservoir, cytoplasme granuleux, mais aussi des caractères divergents : structure nucléaire, nombre de flagelles. Il présente des affinités nettes avec l'organisme signalé simultanément par Hegner et par Wenrich, dans l'intestin de têtards de Rana pipiens à Ithaque (Etat de New-York).

La " species A " de Hegner possède un stigma, des chromatophores, un réservoir avec pharynx et présente trois flagelles. Cette espèce que Wenrich nomme Euglenamorpha Hegneri n'a done que ce dernier caractère pour l'éloigner des Eugléniens connus, alors que tous les autres concordent à l'en rapprocher. Wenrich a rencontré, en outre, associé au précédent, un autre organisme qu'il considère simplement comme une variété de la même espèce ; ce second organisme est incolore, sans stigma, avec gros noyau à caryosome net et 4 à 6 flagelles. La variété incolore présentait des formes de division tandis que la variété verte n'en présentait pas ; celle-ci, en outre, fut cultivée facilement tandis que ce fut impossible pour l'organisme incolore. Cela indique bien une plus grande adaptation à la vie parasitaire du flagellé dépourvu de chlorophylle. Wenrich conclut : "In their typical forms the two varieties are different enough to belong to different species but study of prepared slides has revealed many intermediate conditions which indicate that the green form may transform into the colorless one. "Il nous est difficile, en l'absence des préparations de Wenrich, de conclure à l'identité ou non du flagellé vert et du flagellé incolore; mais il peut paraître bien surprenant qu'en si peu de temps un organisme aussi différencié qu'un Euglénien puisse non seulement perdre sa ehlorophylle et son stigma - ce qui a été observé déjà souvent - mais acquérir 1 à 3 flagelles nouveaux, voir la structure de son noyau se modifier, devenir un parasite obligatoire et perdre la faculté de se développer en culture. Nous pensons plutôt que le flagellé incolore qu'il considère comme une variété est en réalité une espèce distincte de Euglenamorpha Hegneri et cette espèce présenterait - autant qu'on peut en juger, en l'absence de figurés - certaines affinités avec la nôtre. Quoi qu'il en soit, notre espèce constitue le type d'un genre nouveau que nous nommerons Hegneria en l'honneur du savant américain, l'espèce elle-même étant nommée Hegneria leptodactyli.

L'ordre des Euglenidina Büțschli, emend. Delage et Hérouard (=Euglenidea Klebs, = Euglenoidina Blochmann) possèderait ainsi deux groupements nouveaux: Euglenamorpha Hegneri Wenrich devenant le type de la famille des Euglenamorphidæ, comprenant des Euglènes vertes à plus de deux flagelles et notre espèce de- 
venant le type de la famille des Hegneridx, comprenant les Eugléniens incolores à plus de deux flagelles; dans cette famille devra probablement rentrer la " colorless variety " de Wenrich. Entre ces deux familles nouvelles existerait le même parallélisme qu'entre les Euglenidæ et les Astasidæ.

Signalons en terminant que chez les têtards de Leptodactylus ocellatus le rectum présentait une quantité énorme de Wenrichia leptodactyli associés à quelques Trichomonas et Hexamitus sp., tandis que le cœcum et la moitié supérieure du gros intestin présentaient uniquement de nombreuses opalines à deux noyaux (sans doute Zelleriella brasienlis Pinto), et quelques Nyctotherus sp.

Dans les mêmes gîtes existaient d'autres têtards de Leptodactylus sp., de Hyla sp. (3 espèces) et de diverses espèces de Paludicola. Ces têtards présentaient des opalines mais aucun n'hébergeait de Hegneria.

\section{RÉSUMÉ}

Dans des mares des environs de São-Paulo (Brésil) contenant plusieurs espèces de têtards, seuls ceux de Leptodactylus ocellatus nous ont montré abondamment et uniquement dans leur rectum un flagellé ayant 6 ou 7 flagelles égaux, un réservoir avec un pharynx et une cuticule striée.

Ce flagellé que nous nommons Hegneria leptodactyli n. g., n. sp., nous paraît par ses caractères se rattacher aux Euglenidina dont il constituerait une nouvelle famille, les Hegneridæx, à côté de la nouvelle famille des Euglenamorphidæ ayant pour type Euglenamorpha Hegneri Wenrich, 1923.

\section{BibLIOGRAPHIE}

Hegner (E.-W.). - Observations and Experiments on Euglenoidina in the digestive Tract of Frog and Toad Tadpoles. Biological Bull., XLV, N, 3, septembre 1923, p. 162-180.

WeNrich (D.-H.). - Variations in Euglenamorpha Hegneri n. g., n. sp, from the Intestines of Tadpoles. Proced. of the American Soc. of Zoologists (20th Session) in The Anatomical Record, XXIV, 1923, № 6, p. 370-371.

Laboraloire de Parasilologie de la Faculle de médecine de Paris. 